\title{
Modal Analysis of Battery Box Based on ANSYS
}

\author{
Jianong Wang, Xiaoyu Zhao* \\ College of Automotive Engineering, Shanghai University of Engineering and Science, Shanghai, china \\ Email: 594945403@qq.com, "zhaoxiaoyu@sues.edu.cn
}

Received 9 May 2016; accepted 22 May 2016; published 25 May 2016

Copyright @ 2016 by authors and Scientific Research Publishing Inc.

This work is licensed under the Creative Commons Attribution International License (CC BY).

http://creativecommons.org/licenses/by/4.0/

(c) (i) Open Access

\begin{abstract}
At present, the development of the traditional car is more and more troubled by the high cost of environmental pollution and oil prices, many countries have paid increasingly attention to the research and development of electric vehicles. And vehicle battery box, as the heart of the automobile power system, and many difficulties still exist in its research and development. This paper is based on ANSYS. By using the finite element theory, it is to analyze the modal characteristics of the battery box and frequency vibration characteristics. Having a more comprehensive grasp of the dynamic performance of the battery box is the key to solve the new energy automotive research and development of issues.
\end{abstract}

\section{Keywords}

New Energy, Battery Box, Finite Element Theory, Mode Analysis

\section{Introduction}

Starting in 1990s, the research and development of electric vehicles have been valued by the world's major automobile groups, and have received strong support from all countries. After nearly 30 years of research, many important achievements have been made in the field of battery, control system and so on. At present, almost all major automobile groups have the corresponding electric vehicles listed, electric cars will be more and more occupied the car market [1].

In recent years, with the continuous development and progress of science and technology, the development of electric vehicles has made some remarkable achievements. Power battery is not only the direct energy source of pure electric vehicles, but also has a certain impact on the performance of electric vehicles. The battery pack has a direct relationship to the safety performance of the whole vehicle, and as a battery carrier, the cell box, it's

${ }^{*}$ Corresponding author. 
safety and protection of the battery and irreplaceable important role, there for, battery box design has need to consider many factors [2]. Especially, the small pure electric vehicles, as space is limited, and so it must more storage capacity of the battery in the battery box body into, and must also be between vehicle and achieve perfect match. Therefore, design of the battery box has a very high requirement.

\section{Principles in the Modal Analysis}

Modal analysis is a method to study the dynamic characteristics of structure, process analysis, if it is obtained by the finite element method is known for computing modal analysis; if will be collected through the test of system input and output signal through the parameter identification obtained modal parameters, known for experimental modal analysis. Computational modal analysis method has been applied in many product developments, especially in the automotive field, and it is very important to calculate the modal analysis. Modal analysis of the structure is to calculate the natural frequency and vibration mode of the structure, natural frequency and vibration mode are the natural vibration characteristics of the structure under the condition of outside influence [3].

For the finite element analysis of the dynamic response of the structure, the final equilibrium equation of the structure is as follows:

$$
[M]\{\ddot{\delta}\}+[C]\{\dot{\delta}\}+[K]\{\delta\}=\{F(t)\}
$$

Modal analysis is an analysis of the vibration characteristics of the structure under free vibration, and the damping is generally negligible, then it becomes as follows: the free vibration equation:

$$
[M] \ddot{\delta}+[K] \dot{\delta}=0
$$

The free vibration of the elastic body can be transformed into a combination of a series of simple vibrations, which can be used to obtain the natural frequencies of the free vibration and the modes of each order:

$$
\{\delta\}=\left\{\delta_{0}\right\} \sin (\omega x+\phi)
$$

Then put formula (3) into the Equation (2):

$$
\left([K]-\omega^{2}[M]\right)\left\{\delta_{0}\right\}=0
$$

When the structure free vibration, the amplitude of each point in the structure will not be completely zero, so the value of the matrix determinant in the above formula must be zero, so the free vibration frequency equation can be described as follow:

$$
\left|[K]-\omega^{2}[M]\right|=0
$$

Stiffness matrix $[K]$ and mass matrix $[M]$ are all $\mathrm{n}$ matrix, where $\mathrm{n}$ is the number of node degrees of freedom, so the formula is about $\mathrm{n}$ times real coefficient equation of. From the solution of $N$ real root $\omega_{i}^{2}(i=1,2,3, \cdots, n)$, the characteristic value is obtained according to the ascending order as follows:

$$
\omega_{1}^{2} \leq \omega_{2}^{2} \leq \cdots \leq \omega_{n}^{2}
$$

The corresponding vector $\left\{\delta_{0}^{i}\right\}(i=1,2,3, \cdots, n)$ can be solved by the return equation of any $\omega_{i}^{2}$ generation. $\omega_{1}^{2}, \omega_{2}^{2}, \cdots, \omega_{n}^{2}$ are the first order of the structure, the natural frequency of the second order to the $n$ order, Corresponding to $\left\{\delta_{0}^{1}\right\},\left\{\delta_{0}^{2}\right\}, \cdots,\left\{\delta_{0}^{n}\right\}$. Respectively are the structure of the first order, the second order, the first $n$ order of the main type, that is, the natural mode of vibration.

\section{Modal Analysis of Battery Box}

Electric vehicle battery box working in the waste harsh environment, the deformation of some parts will directly affects the electric vehicle battery box normal safe operation. Therefore, it is necessary to analysis the mode of electric vehicle battery box to calculate natural frequencies and mode shapes of the electric vehicle battery box [4]. Due to the finite element modal analysis is a linear analysis, so even if the process of calculation can be defined in some nonlinear factors, in the calculation process will be ignored. In this paper, the modal calculation of 
the box structure of the electric vehicle power battery is selected as the modal calculation module of ANSYS, and there are three ways to extract the modal frequency: Lanczos, Subspace and AMS. Default method of ANSYS is Lanczos method, with more general operation ability, can extract multi order modal calculation results, calculation don't need too high performance computer, very suitable for modal of shell element and solid element mixture model to calculate, the electrical dynamic vehicle battery box structure using ANSYS default Lanczos method [5].

\subsection{Modal Analysis Results}

In this paper, the first 4 order constraint modes of the battery box are calculated, and the inherent frequency is shown in the following Table 1, among them, the constraint of the battery box is the limit of all degrees of freedom around the supporting pin hole.

From the above table, we can draw the following conclusion.

Electric vehicle battery box structure of the natural frequencies are obtained numerically smaller, explain the mechanism of low stiffness, high flexibility, good toughness, can well absorb the seismic energy and the energy produced by the surface of the battery box, the mechanism has high reliability; load corresponding numerical order natural frequency difference is bigger can see differences load corresponding numerical order natural frequency difference is bigger, which indicates that the mechanism of the shape of natural frequency has certain influence.

\subsection{Modal Calculation Results}

In the course of modal calculation, the maximum deformation of each mode is obtained. The maximum amount of deformation that nominal amplitude, it cannot directly said the maximum deformation of the structure of objects, but it reflects the structure of objects in the order type of vibration of maximum deformation position and orientation, here only according to the larger regional deformation were analyzed [6].

Figure 1 shows the mode shapes of the electric vehicle battery box deformation nephogram, the specific deformation trend: first order vibration type mainly for the battery box around $\mathrm{Z}$ axis downward deflection, the maximum deformation is $1.085 \mathrm{~mm}$; second order vibration mode mainly for the battery box around the $\mathrm{Z}$ axis deflection, maximum deformation is $1.120 \mathrm{~mm}$; third order vibration model of the battery box around the $\mathrm{X}$ axis deflection, maximum deformation is $1.238 \mathrm{~mm}$; fourth order vibration type of performance for the battery box around the $\mathrm{Y}$ axis deflection, maximum deformation is $1.325 \mathrm{~mm}$.

When the battery box is in the steady state vibration, the displacement time curve of the center node is shown in Figure 2.

Through the contrast of the battery box vibration type can draw the following conclusions: every order natural frequencies corresponding to different conditions although with different numerical size but the overall deformation tendency is consistent; battery box has larger deformation, indicating the material stiffness is not enough, although the strength of materials to meet the conditions, but in the actual work may cause to the resonance of deformation, when design need to strengthen the stiffness; battery box base structure deformation is high and the rigidity is small, larger resonance failure occurs.

\section{Conclusions}

In this paper, we introduced the flow of finite element modal analysis, and established the finite element model of the dynamic battery box structure, and the results were obtained by the modal analysis of the battery box:

Table 1. Theoretical calculation of the first 4 order modes of the battery box.

\begin{tabular}{ccc}
\hline Order & Frequency/Hz & Vibration position \\
\hline 1 & 27.77 & cover \\
2 & 46.62 & cover \\
3 & 53.30 & surround \\
4 & 61.44 & surround \\
\hline
\end{tabular}


1) The external loads had little effect on the results of modal calculation, and analyze the reasons produced the error, illustrated that the modal parameters only influenced by the fixed parameters of electric vehicle battery box structure's shape and material properties.

2) The base structure of the whole electric vehicle power battery box was not enough, when the resonance phenomenon occurred, it would produce large deformation and damage, which needed to strengthen the rigidity of

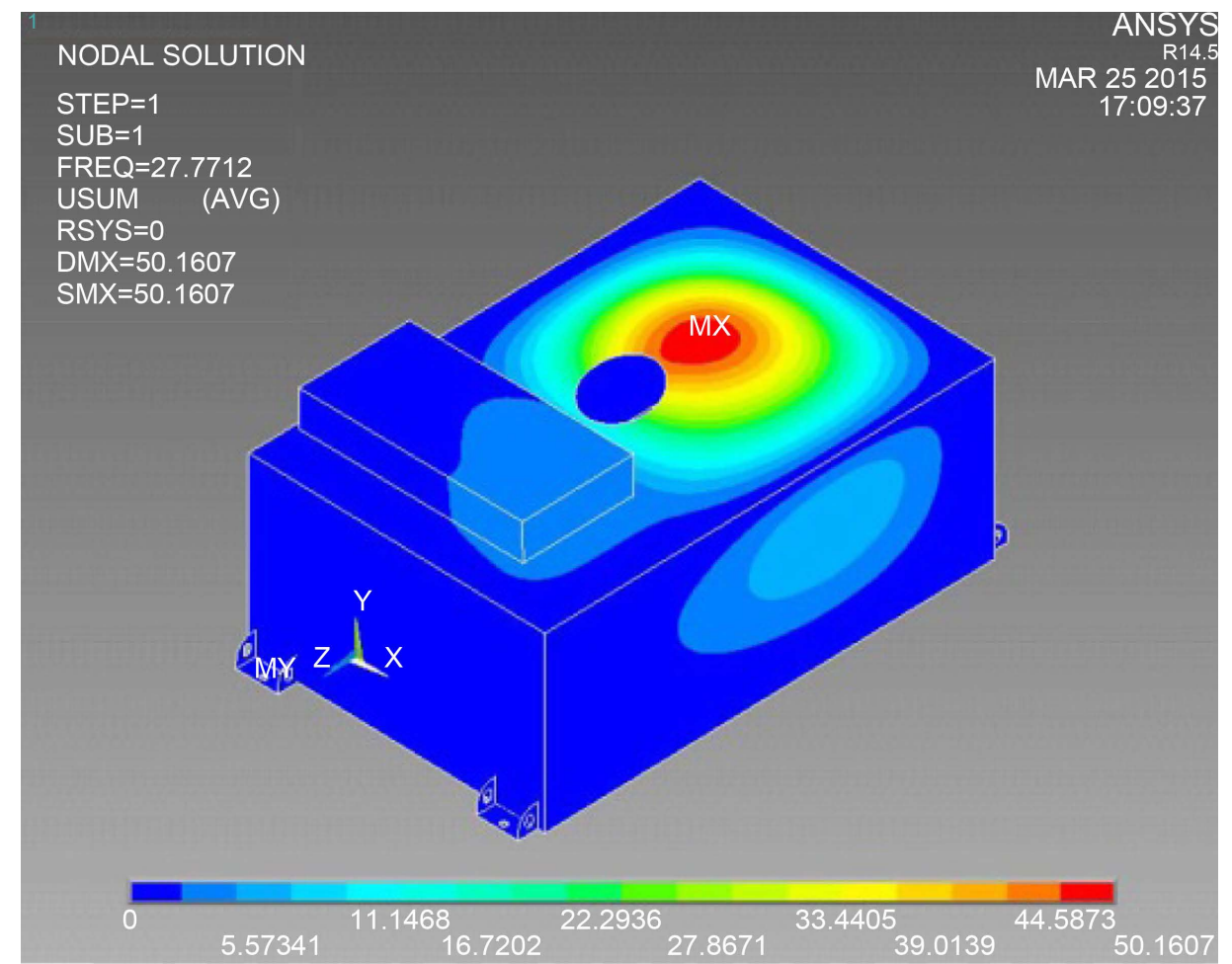

(a) First order mode

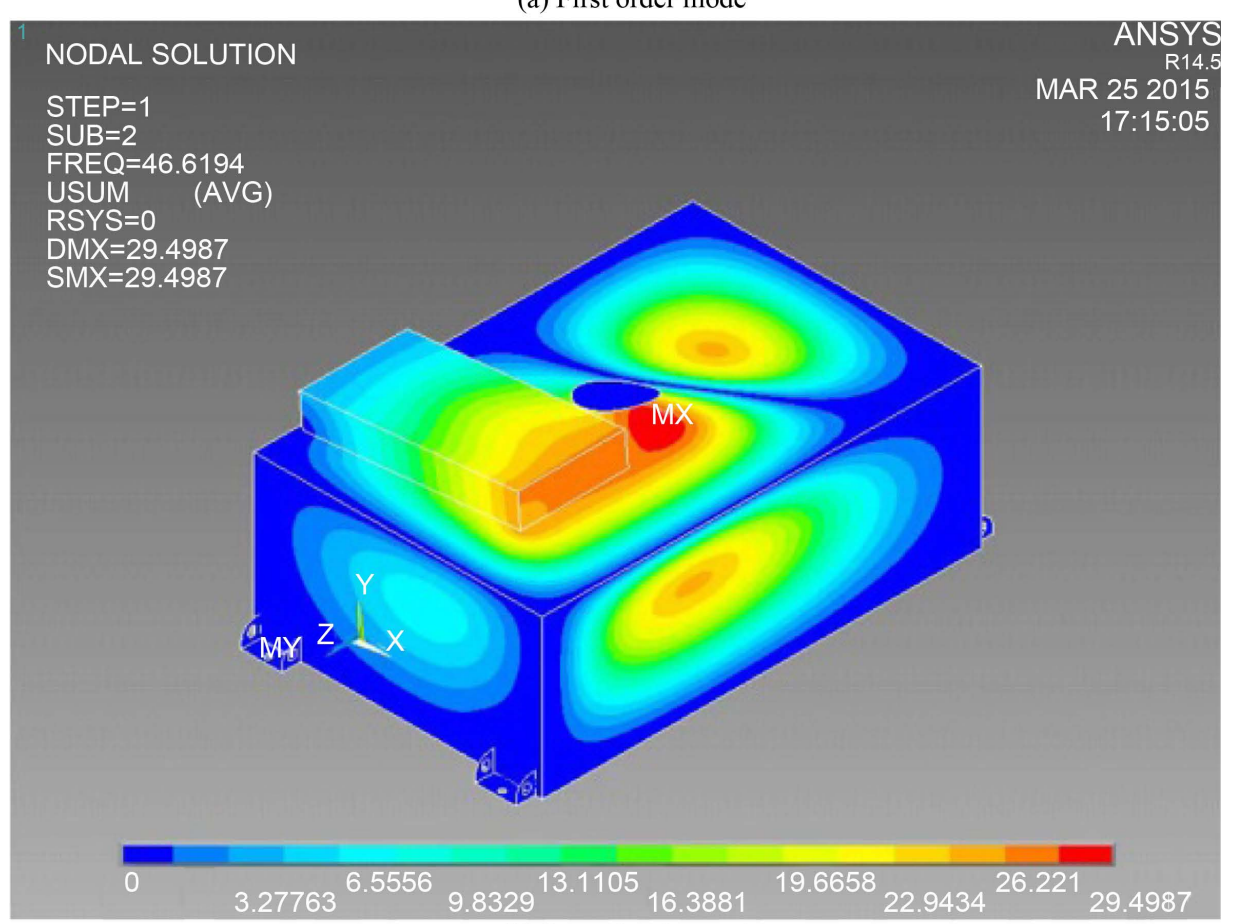

(b) Second order mode 


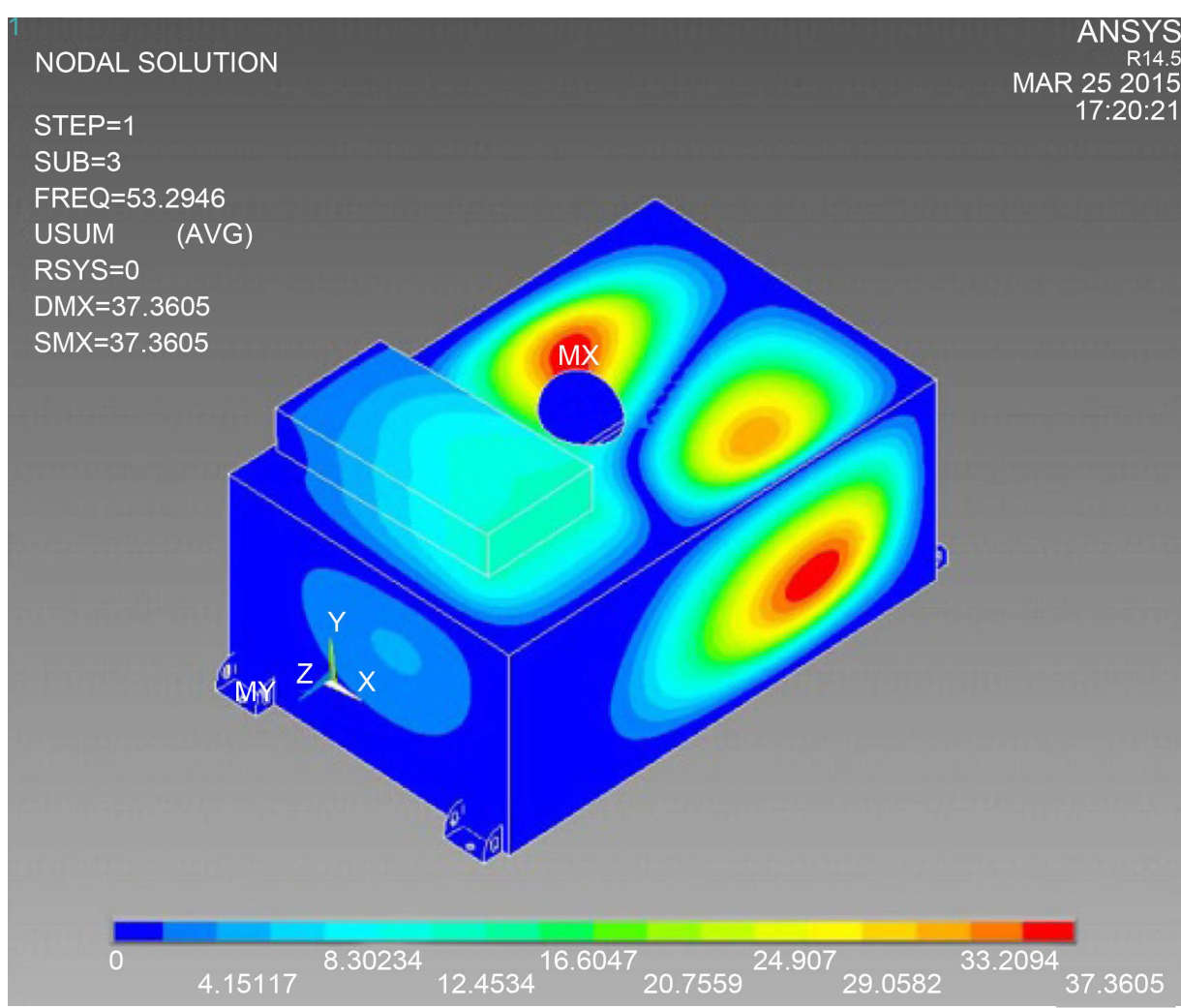

(c) Third order mode

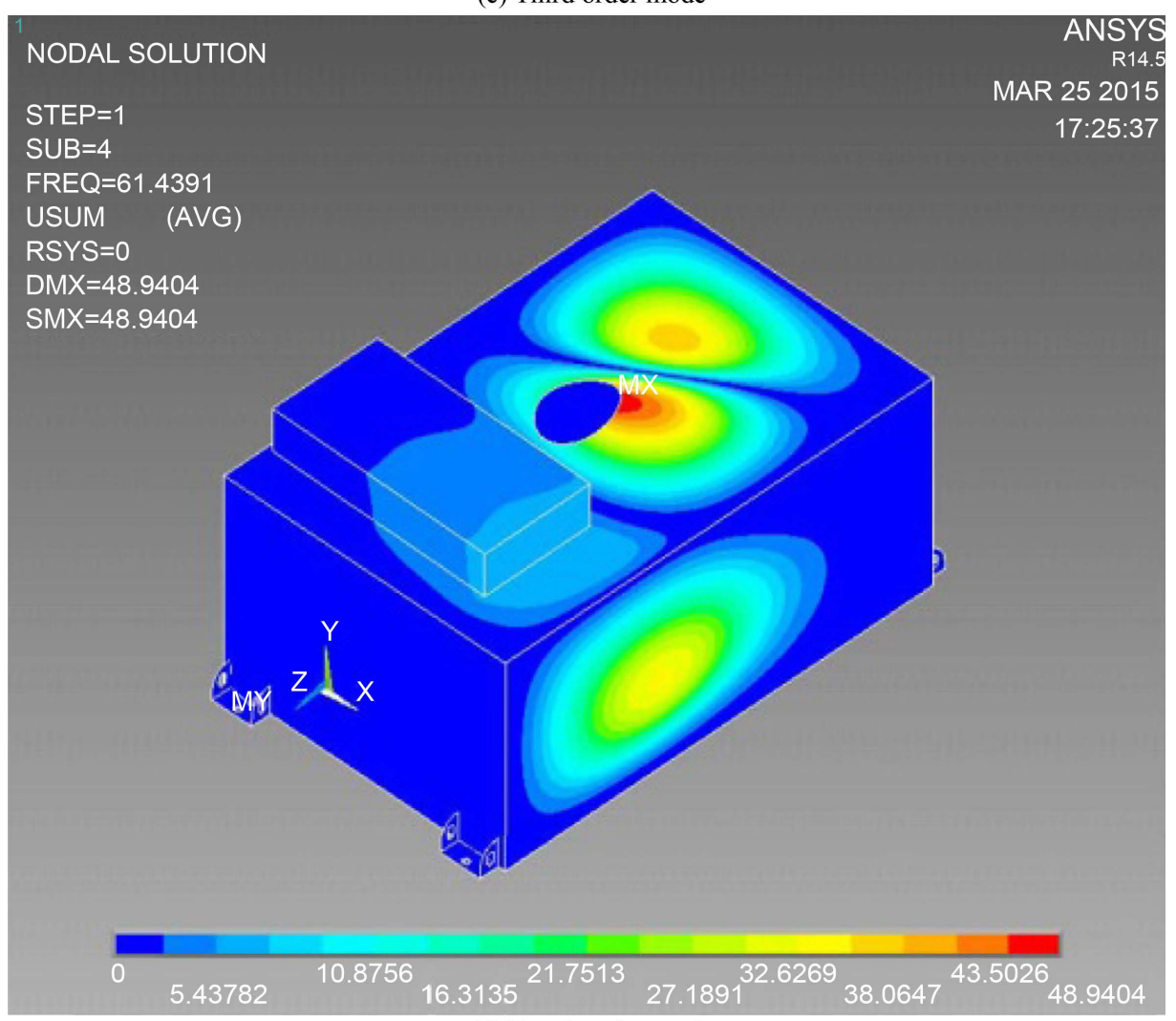

(d) Fourth order mode

Figure 1. Vibration deformation cloud. 


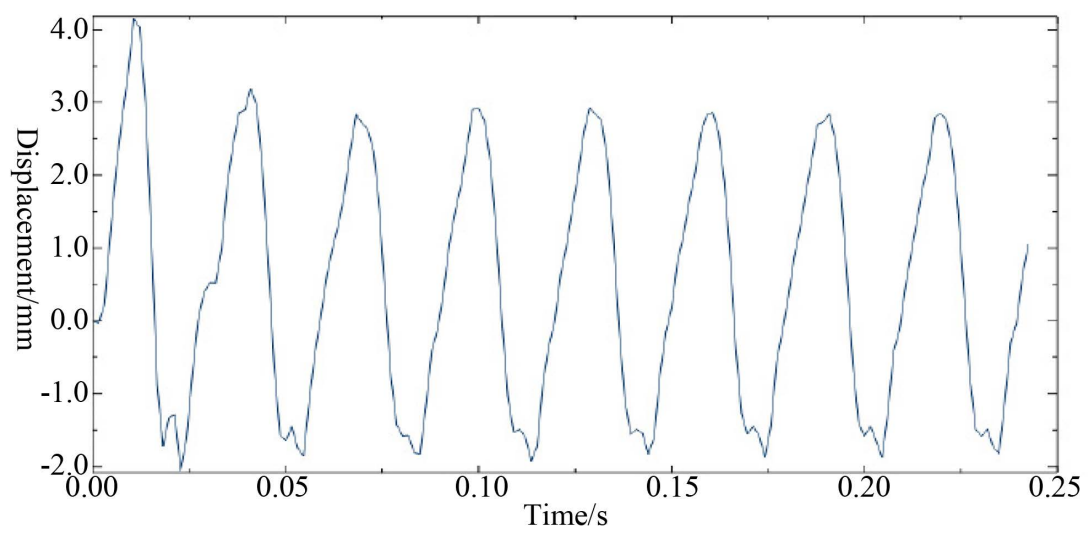

Figure 2. The displacement time curve of the center node of the top cover of the battery box.

these parts in the design process. It provided a reliable basis for the optimization design of the structure of the battery box.

\section{References}

[1] Hu, X. (2015) Finite Element Simulation and Modal Analysis of Power Battery Box for Electric Vehicle. Hunan University, Changsha.

[2] Hartmann, M., Roschitz, M. and Khalil, Z. (2013) Enhanced Battery Pack for Electric Vehicle: Noise, Reduction and Increased Stiffness. Materials Science Forum, 765, 818-822. http://dx.doi.org/10.4028/www.scientific.net/MSF.765.818

[3] Yang, S.J. (2012) Static-Dynamic Analysis and Structural Optimization of Electric Vehicle Battery Box. Hunan University, Changsha.

[4] Wang, B. and Gu, J.W. (2013) Simulation Analysis and Design Optimization of Electric Vehicle Battery Box. New Technology \& New Product.

[5] Jang, G.-W., Yoon, M.-S. and Park, J.H. (2010) Lightweight Flatbed Trailer Design by Using Topology and Thickness Optimization. Structural and Multidisciplinary Optimization, 41, 295-306. http://dx.doi.org/10.1007/s00158-009-0409-X

[6] Ye, Y.H., Shi, Y.X., Cai, N.S., Lee, J.J. and He, X.M. (2012) Electrothermal Modeling and Experimental Validation for Lithium ion Battery. Journal of Power Sources, 199, 227-238. http://dx.doi.org/10.1016/j.jpowsour.2011.10.027 